\title{
Pratiques, la linguistique textuelle et l'analyse de discours, dans le contexte des années 70
}

Pratiques, Text Linguistics and Discourse Analysis, in the Context of the 70s

Jean-Michel Adam

\section{(2) OpenEdition}

Journals

Édition électronique

URL : http://journals.openedition.org/pratiques/2931

DOI : 10.4000/pratiques.2931

ISSN : 2425-2042

Éditeur

Centre de recherche sur les médiations (CREM)

Référence électronique

Jean-Michel Adam, «Pratiques, la linguistique textuelle et l'analyse de discours, dans le contexte des années 70 », Pratiques [En ligne], 169-170 | 2016, mis en ligne le 30 juin 2016, consulté le 14 novembre 2019. URL : http://journals.openedition.org/pratiques/2931; DOI : 10.4000/pratiques.2931

Ce document a été généré automatiquement le 14 novembre 2019.

(c) Tous droits réservés 


\title{
Pratiques, la linguistique textuelle et l'analyse de discours, dans le contexte des années 70
}

Pratiques, Text Linguistics and Discourse Analysis, in the Context of the 70s

\author{
Jean-Michel Adam
}

Je dédie à Jean-François Halté le travail de retour sur un passé commun que j'ai fait à la demande de Caroline Masseron et d'André Petitjean. En me demandant « un bilan des théories des textes et des discours telles qu'elles ont évolué en 40 ans (de la fondation de la revue à aujourd'hui) et dont nous avons pensé qu'elles étaient bénéfiques pour l'enseignement du français ", ils m'ont fixé un programme difficile à remplir. Il m'a toutefois semblé qu'en relisant et repensant les débats théoriques du début des années 70, avec le recul du temps, je parvenais à expliciter le contexte dans lequel, au sein du collectif, nous nous sommes employés à développer une conception du texte et de la diversité des formes de discours à étudier et à enseigner qui est devenue banale aujourd'hui. Je ne traiterai donc qu'un aspect de l'axe 2 du colloque, celui de l'apport de la revue et des membres du collectif aux débats théoriques de la communauté scientifique de langue française. Je laisserai de côté l'analyse de discours (AD) au sens anglo-saxon d'analyse des interactions, tournée vers l'oral, à laquelle J.-F. Halté, en particulier, était plus sensible que d'autres. Je ne dirai rien du modèle holistique de l'interactionnisme socio-discursif développé par Jean-Paul Bronckart, ni de l'évolution de la didactique par rapport aux questions que j'aborderai.

\section{Une génération profondément influencée par É. Benveniste?}

2 Selon moi, quatre grandes figures et propositions théoriques ont été au cœur des débats du collectif de départ de la revue, au début des années 70, à commencer par É. Benveniste. 


\section{1. É. Benveniste et la question du discours}

3 F. de Saussure n'a cessé de s'interroger sur «ce qui sépare » et ce qui unit la langue proprement dite et le «discursif». Il parle d'ailleurs de «langue» et de «langage discursif » (2002 : 95) autant que de «parole ». Prolongeant F. de Saussure, É. Benveniste revient, dans "Sémiologie de la langue ", en 1969, sur la division de la linguistique en deux domaines complémentaires, division qu'il avançait déjà en 1964, dans « Les niveaux de l'analyse linguistique » : «Avec la phrase une limite est franchie, nous entrons dans un nouveau domaine » $(1966: 128)$ et il ajoutait :

La phrase est l'unité du discours. [...] La phrase est une unité, en ce qu'elle est un segment de discours. [...] C'est une unité complète, qui porte à la fois sens et référence: sens parce qu'elle est informée de signification, et référence parce qu'elle se réfère à une situation donnée. (1966:130)

4 Tandis que le domaine «sémiotique » de la langue pouvait continuer à s'appuyer sur la théorie saussurienne du signe, le domaine « sémantique » était appelé à se construire sur la théorie de l'énonciation. La conclusion de l'article de 1969 fixait un programme de travail interrompu par l'accident cérébral qui frappa É. Benveniste en décembre :

En conclusion, il faut dépasser la notion saussurienne du signe comme principe unique, dont dépendraient à la fois la structure et le fonctionnement de la langue. Ce dépassement se fera par deux voies :

- dans l'analyse intra-linguistique, par l'ouverture d'une nouvelle dimension de signifiance, celle du discours, que nous appelons sémantique, désormais distincte de celle qui est liée au signe, et qui sera sémiotique ;

- dans l'analyse translinguistique des textes, des œuvres, par l'élaboration d'une métasémantique qui se construira sur la sémantique de l'énonciation. (1974:66)

Ce programme rompt épistémologiquement avec le principe d'homogénéité structurale allant du phonème, du morphème et du signe jusqu'au texte, principe qui dominera la plupart des conceptions de la textualité, à commencer par l'avant-dernier chapitre des Prolégomènes à une théorie du langage de L. Hjelmslev (1971). R. Barthes est celui qui a le mieux compris l'importance de ce programme.

\subsection{R. Barthes et la translinguistique d'É. Benveniste}

6 Dans un cours inédit de sémiotique littéraire, donné à l'université de Genève en février 1972, R. Barthes parle longuement d'É. Benveniste et il le fait pour les mêmes raisons critiques que celles qui ont poussé de nombreux membres du collectif vers la linguistique: "Je me permets d'insister sur ces catégories linguistiques, dit il, car c'est par là qu'on ébranle le plus nos préjugés ». C'est cet usage de la linguistique comme levier critique qui nous a réunis.

7 Pour R. Barthes, en lisant É Benveniste, on sent, avec le vieux persan, le grec ancien, le vieil irlandais, «le passage de l'étrange et de l'étranger dans le langage ». Ce passage de l'étrange/étranger, R. Barthes dit l'éprouver, quant à lui, dans les textes littéraires : «Le texte fonctionne pour nous comme une sorte de langue étrangère superlative; $[. .$.$] une$ langue contrastante superlative ». C'est tout le sens des notes manuscrites d'É. Benveniste (2011) sur les problèmes posés par la langue de C. Baudelaire :

Өn <Le poète> recrée donc une sémiologie nouvelle, par les assemblages nouveaux et libres de mots. À son tour le lecteur-auditeur se trouve en présence d'un langage 
qui échappe à la convention essentielle du discours. Il doit s'y ajuster, en recréer pour son compte les normes et le sens! (Env. 22, $\mathrm{f}^{\circ} 53$ ). linguistique du discours littéraire à un niveau de très grande exigence :

C'est une langue que le poète est seul à parler, [une langue] qui n'est plus une convention collective, mais expression d'une expérience toute personnelle et unique. Cette langue n'est donc pas connue a priori : celui qui l'entend ou la lit (le rôle de la lecture est immense, peut-être plus important que celui de l'audition) doit s'y former, l'apprendre, et accéder par cet apprentissage à l'intenté du poète. (Env. 22, $\mathrm{f}^{\circ} 48$ )

9 En déclarant, de façon un peu surprenante, qu'É. Benveniste est « le seul linguiste textuel. [...] Il fait la jonction entre la linguistique et la textuologie », R. Barthes pose parfaitement le problème épistémologique : «Si on renonce à décrire l'intégration des phrases dans un texte, on renonce à toute science translinguistique du texte ». Il le disait déjà, en 1966, dans son «Introduction à l'analyse structurale des récits » : « Le discours a ses unités, ses règles, sa "grammaire", au-delà de la phrase et quoique composé uniquement de phrases, le discours doit être naturellement l'objet d'une seconde linguistique " (2002a, p. 831-832). Il revient sur cette "seconde linguistique » dans La linguistique du discours (2002b [1970]) où il réunit les travaux de R. Jakobson et de N. Ruwet sur la poésie, de V. Propp, A. J. Greimas et C. Lévi-Strauss sur les formes narratives du conte et du mythe, et l'analyse du discours de Z. S. Harris sous une même appellation: " une seule sémiotique que nous appellerons, $d u$ moins provisoirement, linguistique $d u$ discours, ou translinguistique (le terme métalinguistique, préférable, étant pris dans un sens différent) » (ibid., p. 611). À la différence d'É. Benveniste, R. Barthes situe le «territoire de la translinguistique » (ibid., p. 612) au-delà de la charnière phrastique et il fait de la question de la segmentation $\mathrm{du}$ discours en unités un préalable épistémologique et méthodologique: "La nécessité de la description intégrative n'est pas seulement épistémologique, elle est aussi opératoire, car c'est d'elle que dépend la segmentation du discours en unités » (ibid., p. 614). Il énonce un ambitieux programme de théorisation des niveaux d'intégration échelonnés de la phrase « jusqu'au moment où le discours s'articule sur la praxis sociale » (ibid., p. 613-614), comme il le disait déjà, en 1966 :

Si l'on essaie d'embrasser l'ensemble d'un récit écrit, on voit qu'il part du plus codé (le niveau phonématique, ou même mérismatique), se détend progressivement jusqu'à la phrase, point extrême de la liberté combinatoire, puis recommence à se tendre, en partant des petits groupes de phrases (micro-séquences), encore très libres, jusqu'aux grandes actions, qui forment un code fort et restreint : la créativité $\mathrm{du}$ récit [...] se situerait ainsi entre deux codes, celui de la linguistique et celui de la translinguistique. (2002a, p. 864)

Cette narratologie linguistique sera tout l'enjeu des travaux menés dans Pratiques sur le récit et sur la description. Par ailleurs, R. Barthes situait la linguistique du discours dans la filiation de la rhétorique en lui assignant la tâche de reprendre à neuf les questions des situations discursives du délibératif, du judiciaire et de l'épidictique, ainsi que de la division du discours « en unités de taille croissante, de la phrase aux grandes parties de la dispositio, en passant par la période et le "morceau" (ekphrasis, descriptio) » (ibid., p. 613). Le processus de linguisticisation de la narratologie et de la rhétorique était ainsi tout tracé. importante : «Ce serait précisément l'une des tâches de la linguistique du discours que de 
fonder une typologie des discours» (2002a, p. 832). C'est ce qu'a entrepris un autre lecteur attentif d'É. Benveniste : Tzvetan Todorov.

\subsection{T. Todorov : les genres et la typologie des discours}

En 1973, dans sa contribution à Qu'est-ce que le structuralisme?, T. Todorov définit le rôle de la poétique comme un rôle «transitoire » de « révélateur des discours» (1973, p. 108). Il déplore le fait que «notre enseignement privilégie encore la littérature, au détriment de tous les autres types de discours » et affirme que "la littérature est impensable en dehors d'une typologie des discours » (ibid.). Il développe cette idée dans « La notion de littérature ", dans le volume d'hommage à É. Benveniste de 1975. Dans cet article, repris dans Les genres du discours en 1978, il expose un programme de recherche auquel nous adhérions, J.-P. Goldenstein et moi, en écrivant Linguistique et discours littéraire (1976) :

À la place de la seule littérature apparaissent maintenant de nombreux types de discours qui méritent au même titre notre attention. Si le choix de notre objet de connaissance n'est pas dicté par de pures raisons idéologiques (qu'il faudrait alors expliciter), nous n'avons plus le droit de nous occuper des seules sous-espèces littéraires, même si notre lieu de travail s'appelle "département de littérature " (française, anglaise, ou russe). Un champ d'études cohérent, pour l'instant partagé impitoyablement entre sémanticiens et littéraires, socio- et ethnolinguistes, philosophes du langage et psychologues, demande donc impérieusement à être reconnu. (1975 : 363-364)

13 En 1978, T. Todorov ajoute une relative à la dernière phrase : « où la poétique cédera sa place à la théorie du discours et à l'analyse de ses genres ». La position de T. Todorov avait l'immense mérite de penser le continu du langage et des discours : «Chaque type de discours qualifié habituellement de littéraire a des "parents" non littéraires qui lui sont plus proches que tout autre type de discours "littéraire" " (1978: 25). Il brisait ainsi la distinction qui traversait alors les travaux allemands sur les textes et les discours, entre les types textuels (Textypen) et les genres textuels (Textsorten), étudiés tous deux par les linguistes, et les genres littéraires (Gattungen) étudiés par les littéraires et privilégiés par les philologues. Cette question des genres ne sera reconnue comme centrale dans l'AD française qu'une dizaine d'années plus tard, quand D. Maingueneau écrira :

Les énoncés relevant de l'AD se présentent en effet non seulement comme des fragments de langue naturelle ou de telle ou telle formation discursive, mais aussi comme des échantillons d'un certain genre de discours. (Maingueneau, 1987, p. 23)

Définissant l'AD comme l'étude du «dispositif d'énonciation qui lie une organisation textuelle et un lieu social déterminés ", D. Maingueneau ajoute quelques années plus tard: "Le discours y est considéré comme activité rapportée à un genre, comme institution discursive » (1996: 8). Dans le Dictionnaire d'analyse du discours, il souligne que « pour la première fois dans l'histoire, la totalité des énoncés d'une société, appréhendée dans la multiplicité de leurs genres, est appelée à devenir objet d'étude » (2002: 45).

C'était le sens du no 7-8 de Pratiques, de décembre 1975, qui mêlait stratégiquement des articles consacrés à la lecture du film, de la bande dessinée, du discours publicitaire et d'un poème des Illuminations d'A. Rimbaud. Quand on survole le traitement de ces questions par la revue, on constate, à côté d'un grand nombre de numéros consacrés au théâtre, à la poésie et au roman ou encore aux Fables de J. de La Fontaine, que de nombreux numéros mêlent stratégiquement les pratiques discursives. Les numéros portent soit sur des genres de textes ( $\left(\right.$ Les genres du récit », $n^{\circ}$ 59, 1988 et « Les discours explicatifs », n 58 , 
1988), soit sur des genres de discours («Genres de la presse écrite », n 94, 1997). Ils abordent la théorie et la pratique des genres : «Didactique des genres » $\left(n^{\circ} 66,1990\right)$ et "Théories et pratiques des genres» ( $\left.n^{\circ} 157-158,2013\right)$. D’autres numéros mettent en avant les types de textes : le $n^{\circ} 51$ (1986) sur «Les textes explicatifs »; le $n^{\circ} 55$ (1987) sur «Les textes descriptifs»; le $n^{\circ} 56$ (1987) sur «Les types de textes»; le nº 62 (1989) «Classer les textes » et le $n^{\circ} 111-112$ (2001) sur « Les textes de consigne ».

Et plusieurs numéros mettent l'accent sur l'activité, en recourant à l'infinitif ou à une substantivisation neutralisante : le $n^{\circ}$ 34, 1982 : «Raconter et décrire »; le $n^{\circ} 99,1998$ : «La description »; le $n^{\circ}$ 109-110, 2001 : «Histoire de la description scolaire »; le $n^{\circ} 28$, 1980 : «Argumenter»; le $n^{\circ} 73,1992$ : «L'argumentation écrite » et le $n^{\circ}$ 96, 1997 : « Enseigner l'argumentation ».

\subsection{La sémiotique textuelle d'A. J. Greimas}

17 En novembre 1976, A. J. Greimas, qui vient de publier Maupassant. La sémiotique du texte: exercices pratiques, donne un entretien très intéressant au collectif de la revue: "Sémiotique narrative et textuelle. Entretien » (1976b). Comme R. Barthes et T. Todorov, A. J. Greimas reconnait que «nous voilà parvenus à la forme actuelle des recherches qui, quel que soit le nom qu'on leur donne, portent sur les possibilités et les moyens de l'analyse des discours » (p. 6), et il ajoute, comme T. Todorov, qu' « on ne peut parler de textes littéraires que dans le cadre général de la théorie du discours » (p. 10). Il définit sa "linguistique discursive» comme une étude «des régularités et des principes d'organisation transphrastiques » (p. 6) qu'il fonde sur une sémantique élargie à tous les systèmes de signes et à laquelle il donne, pour cette raison, le nom de sémiotique. Il considère cette sémiotique comme textuelle: "La sémiotique postule [...] que le texte est sa seule réalité, qu'il est son seul "référent" garantissant l'adéquation de ses constructions métalinguistiques » (p. 10). Poussé à prendre position sur les grammaires de textes et l'interphrastique, A. J. Greimas affirme suivre «avec sympathie les recherches en linguistique textuelle» (p. 7) et il envisage une division du travail :

Il est naturel, il est heureux que des linguistes restent préoccupés [...] par des problèmes d'enchaînement des phrases, en quête d'éléments - tels les anaphoriques et les déictiques - signalant et fondant la continuité discursive au niveau de la surface de texte. C'est un domaine légitime des recherches, parallèle à nos efforts d'établir, à un niveau plus profond, les principes et les règles de l'organisation narrative sous-jacente au déroulement des discours (p. 7).

Dans son Maupassant, A. J. Greimas définissait le «plan isotope du discours » postulé dans Sémantique structurale (1966: 71) en utilisant des termes très proches de la linguistique interphrastique :

L'existence du discours - et non d'une suite de phrases indépendantes - ne peut être affirmée que si l'on peut postuler à la totalité des phrases qui le constituent une isotopie commune, reconnaissable grâce à un faisceau de catégories linguistiques tout au long de son déroulement. Ainsi, nous sommes enclins à penser qu'un discours « logique » doit être supporté par un réseau d'anaphoriques qui, en se renvoyant d'une phrase à l'autre, garantissent sa permanence topique. (1976a, p. 28).

19 A. J. Greimas fondait la cohésion sur le concept transphrastique d'isotopie, largement développé par la suite par F. Rastier, qui en fera un concept textuel dans Sens et textualité (1989), et par M. Arrivé dans «Lire, dé-lire » (1975). Selon J.-C. Coquet, si « les linguistes n'étaient pas en mesure de fournir les modèles discursifs satisfaisants ", c'est "faute 
d'avoir reconnu et analysé le statut sémantique du langage en général et de sa dimension transphrastique en particulier» (1982, p. 34). Cependant, en mettant l'accent sur les structures profondes du sens (carré sémiotique, programmes narratifs, schéma actanciel) et en faisant du principe de narrativité le niveau profond et fondamental d'organisation du discours, la sémiotique ne permettait pas de différencier un conte, d'une nouvelle de G. de Maupassant, d'un roman de G. Bernanos, ni même de la recette de la soupe au pistou. L'«élargissement progressif de la narrativité » est ainsi défini page 8 de l'entretien de Pratiques :

Les discours philosophiques, juridiques, politiques, sans être précisément des «récits», possèdent une dimension narrative qui les organise. Les recherches actuelles que nous poursuivons en groupe [...] tendent à montrer que tout discours est narratif ou plutôt que le terme de "narrativité », devenu trop général et impropre, recouvre en fait une organisation sémiotique du discours qui est sousjacente à sa manifestation linguistique proprement dite.

L'appareil théorique de la sémiotique, à la fois trans-sémiotique et trans-générique, ne permettant pas de rendre compte de la diversité des discours et du fonctionnement de la textualité, mais J.-C. Coquet (1982, p. 31) résume assez bien notre sentiment, à la fin des années 60 et au début des années 70, d'avoir à notre disposition deux types d'approches, l'une plus syntaxique, celle de Z. S. Harris, l'autre plus sémantique, celle d'A. J. Greimas. J.-C. Coquet oppose "le domaine de l'interphrastique», objet de la linguistique du discours, à la «sémantique transphrastique » sémiotique. Avec la sémiotique, on passe en effet d'une "syntaxe» des structures de surface (phrastiques et interphrastiques) aux structures sous-jacentes logicosémantiques transphrastiques. C'est pourtant une autre voie qui allait s'avérer linguistiquement plus féconde.

\section{L'émergence de la linguistique textuelle}

\subsection{L'émergence du texte dans l'analyse du discours}

Dans son article de 1952, traduit en 1969, Z. S. Harris avait l'ambition de présenter « $a$ method for the analisis of connected speech (or writing) ». Ce que F. Dubois-Charlier traduit par " une méthode d'analyse de l'énoncé suivi (écrit ou oral) », mais elle ajoute une relative : "que nous appellerons discours ». Ajout surprenant car Z. S. Harris parle beaucoup plus de texte que de discours : 227 occurrences du vocable text et de ses dérivés (textual et textually) pour 47 occurrences seulement du vocable discourse. À côté des 23 occurrences de la collocation "discourse analysis ", les 24 autres de discourse sont proches du concept de text : les 5 occurrences de la collocation « connected discourse " permettent d'opposer " The successive sentences of a connected discourse " aux "Arbitrary conglomerations of sentences ». Je suis d'accord avec D. Maingueneau pour dire qu'un tel programme, qui ne pense la discursivité qu'en termes de corrélation behaviouriste entre le discours et la réalité sociale, « relèverait aujourd'hui de la linguistique textuelle » (2014, p. 10).

Bien qu'elle mette autant le texte en avant, la méthode harrisienne des classes d'équivalence réduit les phrases complexes à des propositions simples et manipule la surface discursive. La méthode harrisienne rend compte des paragraphes, chapitres et autres «sous-textes à l'intérieur du texte principal » en termes de « sections successives du texte qui contiennent chacune leurs propres classes d'équivalence différentes de celles d'autres sections ». Cette méthode d'établissement des classes d'équivalence par attention aux vocables répétés et associés, n'est pas aussi interphrastique que le dit J.-C. Coquet. 
Elle est proche, en revanche, de l'analyse réticulaire de D. Legallois qui, dans Langages 163 (2006), souligne fort justement que

L'organisation réticulaire du texte est en parfaite congruence avec l'étymologie du mot texte. C'est bien un tissu de phrases enchevêtrées, une trame, une texture, toute une construction et une conception d'un objet complexe que met en évidence l'analyse de la répétition lexicale dans les discours. (Legallois, 2006, p. 70)

L'identification de répétitions et les cooccurrences de vocables ou collocations construites par un texte sont un « aspect décisif de la texture» (Viprey, 2006, p. 73 ; voir également Adam, 2011a).

La même année 1969 que la traduction de l'article de Z. S. Harris, dans le $\mathrm{n}^{\circ} 13$ de Langages fondateur de l'AD française, paraissent L'Archéologie du savoir de M. Foucault et Analyse automatique du discours de M. Pêcheux. M. Foucault, qui parlait plus volontiers d' « analyse historique du discours » $(1969$, p. 36) et d' « analyse des formations discursives » (p. 100), ne faisait porter cette analyse ni sur le style, ni sur l'enchaînement des phrases, laissant ainsi - selon son expression - «en pointillé la mise en place finale du texte »(ibid.). M. Pêcheux allait dans le même sens :

Il est impossible d'analyser un discours comme un texte, c'est-à-dire comme une séquence linguistique fermée sur elle-même, mais [...] il est nécessaire de le référer à l'ensemble des discours possibles à partir d'un état défini des conditions de production. (1990 [1969], p. 115).

M. Pêcheux et C. Fuchs réaffirment cette position, en 1973, en excluant l'idée même d'analyse d'un seul texte, comme le fait Z. S. Harris (1973, p. 14). Ce que formule autrement G. Provost-Chauveau :

L'analyse du discours suppose la mise ensemble de plusieurs textes. [...] Le texteexemple de discours - n'est jamais réellement clos, il est continuation, d'une part, et inachèvement, de l'autre. Il n'existe comme texte que par ce qui le précède et le constitue, c'est-à-dire en fonction d'autres discours. (1971, p. 19)

26 En 1975, dans un long article de Langages 37, M. Pêcheux et C. Fuchs, à l'occasion d'une critique de «la domination théorique de la phrase» $(1975$, p. 44$)$, font une des rares allusions de l'AD à la linguistique textuelle (LT) :

Le développement actuel des recherches linguistiques, et le lien qui s'établit progressivement entre l'inter-phrase et la paraphrase dans le cadre des "linguistiques du texte » qui se font jour, permettent de penser que la linguistique est aujourd'hui sur la voie de la solution à ce problème, qui, comme nous l'avons dit, en commande un grand nombre d'autres (Pêcheux \& Fuchs, 1975, p. 44)

À partir du colloque Matérialités discursives de 1980, il sera de plus en plus question de " séquentialité intradiscursive » et d' " analyse linguistique du "fil du discours" ". Dans la table ronde de ce colloque, A. Culioli n'hésite pas à voir dans les diverses formes et tendances de l'AD « une manière de relayer l'explication de textes en tant qu'exercice scolaire ». Nous ne disions pas autre chose dans Pratiques depuis les premiers numéros!

Il faudra attendre 1985 pour que, faisant allusion aux travaux de D. Slakta, C. Fuchs parle de lacune des travaux existants à propos de la question du texte et déplore que les études menées sur l'ambigüité et la paraphrase ne s'intéressent qu' « aux ambiguïtés de phrases isolées et aux relations de synonymie entre phrases prises deux à deux, sans considération de contexte plus vaste. [...] L'on ne dispose pas d'études systématiques sur l'ambiguité et la paraphrase au niveau du texte» (1985, p. 20). Et elle conclut: «La limitation à la phrase, dans ce domaine de la constitution de la signification, apparaît comme une restriction dommageable » (p. 21). 
la position que D. Maingueneau défendait depuis 1976. La prise en compte de la surface discursive et l'analyse d'énoncés suivis lui paraissaient «inéluctables » (1976, p. 152) et il voyait même dans la question non posée du texte une « lacune grave » de l'AD (ibid.). En 1991, il qualifie la grande méfiance de l'AD à l'égard des formes de continuité discursive de "présupposé erroné » (1991, p. 207). Sa position est très claire : «L'AD a affaire à des textes. Il lui faut donc prendre acte du fait qu'un texte n'est pas une suite aléatoire de phrases, que ses constituants obéissent à certaines règles d'enchaînement " (ibid.). D'où son intérêt, manifeste déjà dans son livre de 1976 (p.151-182), pour la «cohérence textuelle» et pour les grammaires de texte. Ne partageant pas l'ambition grammairienne (présente déjà chez Hjelmslev, 1971: 27) de générer tous les textes bien formés, « concevables et théoriquement possibles » d'une langue donnée, D. Maingueneau souligne l'incompatibilité manifeste entre les options épistémologiques des grammaires textuelles (GT) d'orientation générativiste et l'AD, mais cela ne l'empêche pas de trouver qu'elles décrivent des phénomènes négligés par la linguistique et dont l'AD ne peut se passer.

En langue française, au début des années 70, on ne pouvait guère lire que deux articles traduits dans un numéro de Langages sur «La grammaire générative en pays de langue allemande », coordonné par D. Clément et B. Grünig :

- Bourdin, J.-F. \& Duhem, P. (1972). « La grammaire de texte en pays de langue allemande ». Langages 26, p. 59-74;

- Lang, E. (1972). "Quand une "grammaire de texte" est-elle plus adéquate qu'une "grammaire de phrase" ? ». Langages 26, p. 75-80.

Les premiers articles en français de T. A. van Dijk s'inscrivaient explicitement dans le prolongement du courant dominant de la théorie générative :

- (1972). «Aspects d'une Théorie Générative du Texte Poétique ». In : Greimas, A.-J. et al., Essais de sémiotique poétique. Paris : Larousse, p. 180-206.

- (1973a). « Modèles génératifs en théorie littéraire ». In : Bouazis, C. et al., Essais de la théorie du texte. Paris : Galilée, p. 79-99.

- (1973b). « Grammaires textuelles et structures narratives ». In : Chabrol, C. (éd.), Sémiotique narrative et textuelle. Paris : Larousse, p. 177-207.

Deux traductions de l'allemand, moins proches de la grammaire générative, suivront: Linguistique et sémiologie $\mathrm{n}^{\circ} 5,1978$, consacré à la Textlinguistik; et, sous l'impulsion de M. Charolles, la traduction par J.-P. Colin du livre de H. Rück sur la didactique du français langue étrangère (1978).

En 1976, M. Charolles avait déjà engagé une critique de fond des GT, dans « Note de lecture de N. Ruwet, Parallélismes et déviations en poésie " (Pratiques 10, 1976, 105-112). L'article de N. Ruwet (1975) pointait les limites de la théorie du discours de Z. S. Harris en s'appuyant sur un article d'I. Bellet (1970). M. Charolles reprochait à N. Ruwet et à I. Bellert de ne pas

tenir compte de contraintes globales pesant, non plus sur l'articulation des phrases, mais sur l'articulation des séquences de phrases. D'où la nécessité de prévoir une théorie du texte susceptible de rendre compte, à la fois, de la cohérence séquentielle (ou micro-structurelle) et de la cohérence textuelle proprement dite (ou macro-structurelle). (1976, p. 110)

Proches des travaux de W. Kintsch \& T. A. van Dijk (1975, 1981-1982), nous montrerons, M. Charolles et moi, que les dérivations de macropropositions (au sens de regroupements de propositions) sont régies par des impératifs moins linguistiques (au sens étroit du 
terme) que cognitifs : limites de la mémoire de travail et recours à des connaissances stockées en mémoire à long terme. Des patrons génériques, des scripts, des connaissances prototypées de suites narratives, argumentatives, etc. (qui seront au centre de ma théorie des séquences textuelles), induisent une certaine structuration hiérarchique des contenus, mais ces connaissances n'ont en rien la nature de règles grammaticalisables dans un modèle de GT. M. Charolles le redit dans «Analyse de discours, grammaire de texte et approche grammaticale des faits de textualité » $(1989$, p. 12) :

Il devient évident qu'une grammaire de texte fondée sur l'idée naïve qu'il existe un critère de bonne formation textuelle comparable à celui de grammaticalité pour la phrase est condamnée à formuler des règles qui seront mises en défaut à la moindre occasion.

Ce constat rend plus ou moins obsolète tout projet de grammaire de texte. Il n'interdit pas cependant que l'on essaie de rendre compte des opérations mises en œuvre par les sujets pour calculer la cohérence d'une séquence d'énoncés, puisque, cohérence il doit (par principe) y avoir. D'où l'évolution des grammaires de texte vers des modèles dits procéduraux censés décrire les calculs effectués par les interprétants pour résoudre la cohérence d'une séquence.

On sent ici l'influence des thèses de H. P. Grice (1975), revues par D. Sperber \& D. Wilson (1986) et J. Moeschler \& A. Reboul (1998). La conception du discours et du texte est, chez M. Charolles, très nettement pragmatique (voir également sur ce point Lundquist, 1999).

D. Maingueneau avait l'immense mérite, dès 1976, de mettre les développements en cours des GT en rapport avec les travaux sur le récit et sur l'argumentation. Position originale pour l'époque car la narratologie avait tendance à occuper tout le terrain du transphrastique. C'est ainsi que nous avons été quelques-uns à nous intéresser à la logique naturelle développée dans le Centre de recherches sémiologiques de Neuchâtel dirigé par Jean-Blaise Grize et à ses travaux sur l'argumentation et l'explication. D. Maingueneau a également perçu très tôt l'importance des travaux d'O. Ducrot sur les connecteurs. Dans Nouvelles tendances en analyse de discours (1987), il déplace les propositions des Mots $d u$ discours (1980) vers une problématique textuelle qui n'a jamais été au centre de la théorie d'O. Ducrot. C'est la position que je défends également, dans Pratiques 43: «Des mots au discours: l'exemple des principaux connecteurs" (1984) et dans mes Éléments de linguistique textuelle (1990).

\subsection{L'émergence de la linguistique textuelle dans Pratiques}

Sans se positionner collectivement dans le champ de l'AD, Pratiques a joué un rôle important dans le développement de la linguistique textuelle (LT) en France. Tout commence en 1974 par un article de J.-F. Halté : «La grammaire et les textes ». Article prolongé, l'année suivante, par B. Combettes et J. Fresson : «Quelques éléments pour une linguistique textuelle» (1975). Trois numéros s'inscrivent très clairement dans cette perspective: le $n^{\circ} 10$ (1976) «Enjeux des textes», les n 11-12 (1976) et 14 (mars 1977) consacrés au « Récit » et le $n^{\circ} 13$ «Textes-linguistique » de janvier 1977. Dans une grosse synthèse de Pratiques 11-12: "Grammaire de texte - Théorie du discours - Narrativité " (1976b, p. 133-154), M. Charolles complète sa note de lecture du $n^{\circ} 10$. Dans ce même numéro, j'attire l'attention sur Tempus d'H. Weinrich : «Langue et texte : imparfait/passé simple» (Pratiques 10, 1976). Traduit en 1973, le livre de celui qui allait donner le premier cours de LT au Collège de France en 1990 ne connaissait qu'un très faible écho dans la 
communauté des linguistes français. La LT d'H. Weinrich présentait pourtant l'intérêt de définir le texte comme un système réticulaire de « relations d'interdépendance »:

Les formes temporelles viennent d'abord à nous - et nous reviennent - à travers des textes. C'est là qu'elles dessinent, avec d'autres signes, et aussi avec d'autres temps, un complexe de déterminations, un réseau de valeurs textuelles. (1973, p. 13).

Nous étions quelques-uns à nous intéresser à la thèse d'H. Weinrich sur la «mise en relief » et à l'analyse textuelle de l'opposition de l'imparfait et du passé simple. J. Simonin-Grumbach, auteure d'un article très important sur la typologisation énonciative des discours (1975), développe dans Pratiques 13 (1977) une critique d'H. Weinrich à la lumière des thèses d'A. Culioli : «Linguistique textuelle et études des textes littéraires. À propos de Le Temps d'H. Weinrich ». Dans Pratiques 100, je suis revenu, avec F. Revaz et G. Lugrin, sur cette question en opérant une critique des propositions d'É. Benveniste et d'H. Weinrich : « Pour en finir avec le couple récit/discours » (1998).

Dans Pratiques 10, J.-P. Goldenstein tirait les conséquences de la grammaire de texte pour la didactique de l'expression écrite: "Une grammaire de texte pour la composition française » (1976). J.-F. Halté et Petitjean donneront également une suite d'articles consacrés à l'intérêt de la GT pour ce qui concerne la planification des textes écrits en situation scolaire. L'équipe de Pratiques a largement participé, en 1978, au n 38 de Langue française : « Enseignement du récit et cohérence du texte », coordonné par M. Charolles et J. Peytard. Je cite juste les contributions des membres du collectif, très directement inscrites dans la linguistique textuelle:

- Charolles, M. (1978). «Introduction aux problèmes de la cohérence des textes ». Langue française 38, p. 7-41.

- Halté, J.-F. \& Petitjean, A. (1978). « Lire et écrire en situation scolaire ». Langue française 38, p. 58-73.

- Combettes, B. (1978). «Thématisation et progression thématique dans les récits d'enfants ». Langue française 38, p. 74-86.

- Adam, J.-M. (1978). «La cohésion des séquences de propositions dans la macro-structure narrative ». Langue française 38, p. 101-117.

Dans Pratiques 13, B. Combettes présente les thèses du second cercle de Prague (Firbas, 1964 et Daneš, 1974) : «Ordre des éléments de la phrase et linguistique du texte » (1977) tandis que je reviens, dans «Ordre du texte, ordre du discours» (1977), sur un article de D. Slakta (1975). Les thèses pragoises sur les progressions thématiques nous apparaissaient comme une alternative aux GT générativistes et un moyen intéressant de rendre compte de l'interphrastique. B. Combettes reviendra sur cette question dans un article épistémologiquement important de Pratiques 77: «Grammaire de phrase, grammaire de texte: le cas des progressions thématiques " (1993) et dans son livre Pour une grammaire textuelle. La progression thématique (1983). Ses travaux largement reconnus donneront deux livres: L'organisation du texte (1992) et Les constructions détachées (1998) ainsi qu'un article épistémologiquement limpide : «Questions de méthode et de contenu en linguistique du texte » (1992), dans lequel il explique que la cohésion et la cohérence du texte n'étant pas les résultantes de faits de grammaticalité, il en résulte une «distorsion », un décalage entre les catégories de la grammaire et celles de la LT. Les concepts utilisés en grammaire phrastique :

possèdent leur propre utilité, dans leur ordre, mais ne peuvent être "réutilisés » tels quels, dans une problématique qui s'attache à un autre domaine que le leur. [... ]. Même si des relations, des interactions, peuvent être observées entre les deux domaines (phrase et texte), des catégories fondées sur des caractéristiques 
purement grammaticales ne peuvent être considérées comme des notions fondamentales pertinentes en ce qui concerne la cohérence du texte. (1992, p. 113-114) contribution décisive à la distinction des plans ou niveaux d'organisation textuelle : « Les plans d'organisation textuelle: périodes, chaînes, portées et séquences » (Pratiques 57, 1988, 3-13); propositions qu'il révisera dans deux articles publiés ailleurs : «Les plans d'organisation du discours et leurs interactions » (1993) et «L'encadrement du discours. Univers, champs, domaines et espaces » (1997). Il montre que les solidarités syntaxiques entre unités de la langue n'ont qu'une portée très limitée. Dès que l'on passe le seuil du syntagme et du noyau de la phrase de base, d'autres systèmes de connexions apparaissent, qui ne reposent pas sur des critères morphosyntaxiques, mais sur des marques et des instructions relationnelles ascendantes et descendantes de portée plus ou moins lointaine. Ces marques de connexion ont "pour fonction conventionnelle de signaler au destinataire que telle ou telle unité doit être comprise comme entretenant telle relation avec telle ou telle autre " (Charolles, 1993, p. 311). La tâche de la LT est de définir la portée et de catégoriser ces marques d'ouverture ou de fermeture des segments textuels plus ou moins longs. Ces marques instructionnelles n'entrant pas dans des schémas grammaticalement préétablis, les domaines textuel et morphosyntaxique sont différents et assez largement indépendants.

À la suite de l'« approche textuelle de la grammaire » de T. Givón (1998) et des nouvelles approches fonctionnalistes, M. Charolles parle plutôt aujourd'hui d'un continuum entre la phrase et le texte/discours. Selon T. Givón, rappelle M. Charolles dans « De la phrase au discours : quelles relations? ", "si l'on veut comprendre quoi que ce soit à la grammaire, il faut l'envisager comme un dispositif destiné à guider la compréhension des échanges communicationnels qui sont de nature discursive ». Pour Givón,

la grammaire est un instrument de codage commun aux informations relevant de la sémantique propositionnelle (phrases simples) et de la cohérence pragmatique discursive (discours). [...] Le gros du codage grammatical se déploie dans le domaine de la pragmatique discursive, signalant ainsi la cohérence de l'information véhiculée dans son contexte situationnel, inter-phrastique et culturel. (1998, p. 269)

Dans ce même article, M. Charolles reconnait qu'il est tout à fait normal que les schémas de textes (pour moi les séquences, mais aussi l'organisation en paragraphes et les plans de textes) et que les types de discours (pour moi les genres de textes et les genres de discours) guident l'élaboration de structures plus complexes que les schémas phrastiques.

\subsection{La stabilisation de la linguistique du texte et du discours}

La stabilisation de la place de la LT dans la linguistique de langue française est marquée, d'une part, par l'apparition d'ouvrages qui prennent ouvertement le texte comme objet et, d'autre part, par l'introduction du texte dans les manuels de linguistique et de grammaire. 

approches herméneutique et philosophiques de P. Ricœur (1986) et J. Greisch (1987) et les livres de F. Rastier $(1989,2001)$, de S. Stati (1990), de D. Apothéloz (1995), de J. Gardes Tamine et M.-A. Pellizza (1998), de S. Carter-Thomas (2000), de F. Jacques $(2002,2007)$, les participations de membres du collectif de Pratiques au développement du domaine ont été déterminantes : B. Combettes (1983, 1992, 1998), B. Combettes \& R. Tomassone (1988), J.M. Adam \& A. Petitjean (1989), J.-M. Adam (1985, 1990, 1992, 2005, 2011), M. Charolles (2002).

Pour la manuélisation, le dernier chapitre de la Grammaire méthodique du français de M. Riegel, J.-C. Pellat et R. Rioul passe de 20 pages sur « La structuration du texte » en 1994 à 47 pages dans l'édition 2009 (p. 1017-1064) et le titre du chapitre est significativement modifié: "Texte et discours». On peut aussi citer le dernier chapitre, consacré au "Texte», du manuel d'initiation à la linguistique d'O. Soutet (1995, repris dans la collection Quadrige en 2005, p. 323-346). Dans ce manuel d'initiation à la linguistique, 0 . Soutet adopte la ligne que B. Combettes, M. Charolles et moi avons soutenue dans Pratiques:

On ne saurait décomposer le texte en phrases comme on peut décomposer la phrase en syntagme, le syntagme en morphèmes et le morphème en phonème - ce qui signifie que, dans le cas particulier du texte, le rapport du tout à la partie ne relève pas du même type de prévisibilité que celui qui existe entre chacune des unités subphrastiques et leurs constituants immédiats. (1995, p. 325)

Dans leur livre sur Les grandes théories de la linguistique M.-A. Paveau et G.-É. Sarfati situent la LT dans les «linguistiques discursives» (2003, p. 184-194). Et, dans sa dernière présentation de l'AD, D. Maingueneau accorde à la LT et à la question du texte une place à la fois plus importante et plus claire qu'avant, en écrivant que

à partir des années 1960 - c'est-à-dire en même temps que l'analyse du discours -, s'est développée une nouvelle discipline, la linguistique textuelle, qui, visant à dégager des régularités au-delà de la phrase, fournissait aux analystes du discours des instruments précieux pour appréhender la structuration des textes. (2014, p. 12)

Il reconnait la double organisation transphrastique des discours par les genres de discours et par « les règles, transversales aux genres, qui gouvernent un récit, un dialogue, une argumentation, une explication... » (ibid., p. 19). Revenant sur ces « règles transversales aux genres ", il mentionne les typologies énonciatives et les typologies "fondées sur les modes d'organisation transphrastiques, la manière dont sont structurés les textes » et il rapproche fort justement ma théorie des séquences des pré-genres de J. M. Swales (1990), «soulignant par là que ce sont en quelque sorte des matériaux pour les genres de discours proprement dits » (2014, p. 102).

\section{Pour conclure}

Ce rapide parcours, qui nous a menés des années 70 du siècle passé à aujourd'hui, avait pour but de contextualiser une partie des travaux menés au sein de Pratiques dans le domaine de la théorie et de la pratique des textes et des discours. Ces pages font, je l'espère, ressortir à la fois le bouillonnement intellectuel des années 60-70 (des 20 ans de certains d'entre nous) et la richesse du dialogue continu que nous avons pu mener, pendant près de 40 ans, entre nous et en dehors du collectif. Sans le soutien critique des propositions des uns et des autres, sans le travail de la pensée autour de thématiques qui

Pratiques, $169-170 \mid 2016$ 
nous réunissaient et que je viens de rappeler, le développement d'une linguistique textuelle en langue française n'aurait pas été possible, tant le contexte institutionnel y était peu favorable, en France en particulier. La revue a joué un rôle de diffusion d'idées et de recherches qui n'étaient alors pas académiquement reconnues. Elle a joué aussi un rôle de stimulation de questionnements en prise sur la demande sociale (celle de l'école d'abord, bien sûr). Cette linguistique textuelle « à la française » apparait ainsi aujourd'hui comme doublement axée sur la question théorique des problèmes du texte et du faire texte (Adam, 2014, 2015) et sur la collaboration avec les champs disciplinaires et domaines d'application proches que sont l'analyse de discours, la stylistique et la traduction, mais aussi en prise sur les didactiques de l'écrit et de la lecture des textes.

\section{BIBLIOGRAPHIE}

ADAM, J.-M. (1976). « Langue et texte : imparfait/passé simple ». Pratiques 10, p. 49-68.

- (1977). «Ordre du texte, ordre du discours ». Pratiques 13, p. 103-111.

- (1978). « La cohésion des séquences de propositions dans la macro-structure narrative ».

Langue Française 38, p. 101-117.

- (1984). « Des mots au discours : l'exemple des principaux connecteurs ». Pratiques 43,

p. 107-122.

- (1985) [1994]. Le texte narratif. Paris : Nathan.

- (1990). Éléments de linguistique textuelle. Bruxelles : Mardaga.

- (2001). « Barthes en 1970 : de la translinguistique à la déconstruction ». In : Boissinot, A. et al.

(éds), Littérature et sciences humaines. Paris : Les Belles-Lettres/CRTH de l'université de CergyPontoise, p. 125-148.

- (2010). «L'émergence de la linguistique textuelle en France (1975-2010). Parcours

bibliographique en 100 titres ", Verbum 32, 2, p. 237-261.

- (2011a) [2005, 2008]. La linguistique textuelle. Introduction à l'analyse textuelle des discours. Paris :

Armand Colin.

- (2011b) [1992]. Les textes : types et prototypes. Paris : Armand Colin.

- (2011c). «Le programme de la "translinguistique des textes, des œuvres" et sa réception au seuil des années 1970 ». In : Brunet, É. \& Mahrer, R. (éds.), Relire Benveniste. Réceptions actuelles des Problèmes de linguistique générale. Louvain-la-Neuve : Academia, p. 123-147.

- (2011d). Genres de récits. Narrativité et généricité des textes. Louvain-la-Neuve : Academia.

- (2012). «Les problèmes du discours poétique de Benveniste. Un parcours de lecture ", Semen

33, p. 25-54.

- (2014). Problèmes du texte. Leçons d'Aarhus, Pré-Publications nº 200/2013. Aarhus Universitet :

Fransk institut for æstetik og kommunikation. En ligne : http://dac.au.dk/forskning/tidsskrifter/ prepublications.

- (2014). « Texte et intra-texte : retour sur un rendez-vous manqué de l'analyse de discours et de la linguistique textuelle ». In : $4^{e}$ Congrès mondial de linguistique française. En ligne : http://dx.doi .org/10.1051/shsconf/20140801395.

- (éd.) (2015). Faire texte. Frontières textuelles et opérations de textualisation. Besançon : Presses universitaires de Franche-Comté. 
ADAM, J.-M. \& GoldENSTEIN, J.-P. (1976). « Vers une grammaire de texte ». In : Adam, J.-M., Linguistique et discours littéraire. Théorie et pratique des textes. Paris : Larousse, p. 185-250.

ADAM, J.-M., LUGRIN, G. \& REVAZ, F. (1998). « Pour en finir avec le couple récit/discours ». Pratiques 100, p. 81-98.

ADAM, J.-M. \& PETITJEAN, A. (1989). Le texte descriptif. Paris : Nathan.

APOTHÉLOZ, D. (1995). Rôle et fonctionnement de l'anaphore dans la dynamique textuelle. Genève : Droz. ARRIVÉE, M. (1975). « Lire, dé-lire ». Pratiques 7-8, p. 7-20.

BARTHES, R. (2002a) [1966]. « Introduction à l'analyse structurale des récits ». In : Barthes, R., Euvres complètes II. Paris : Seuil, p. 828-865.

- (2002b) [1970]. « La linguistique du discours ». In : Barthes, R., CEuvres complètes III. Paris : Seuil, p. 611-616.

BELLERT, I. (1970). « On a condition of the coherence of texts ». Sémiotique 2, 4, p. 335-363.

BENVENISTE, É. (1966) [1964], « Les niveaux de l'analyse linguistique ». In : Proceedings of the Ninth International Congress of Linguists, La Haye : Mouton, ch. X.

- (1969). «Sémiologie de langue (1) ». Semiotica. Revue de l'Association internationale de sémiotique 1, 1, p. 1-12.

- (1974). Problèmes de linguistique générale II. Paris : Gallimard.

- (2011). Baudelaire. Éd. établie par C. Laplantine. Limoges : Lambert-Lucas.

BOURDIN, J.-F. \& DUHEM, P. (1972). « La grammaire de texte en pays de langue allemande », Langages 26, p. 59-74;

BRONCKART, J.-P. (1996). Activités langagières, textes et discours. Lausanne/Paris : Delachaux/Niestlé. CARTER-THOMAS, S. (2000). La cohérence textuelle. Pour une nouvelle pédagogie de l'écrit. Paris :

L'Harmattan.

Charaudeau, P. \& maingueneau, D. (éds) (2002). Dictionnaire d'analyse du discours. Paris : Seuil.

CHARolles, M. (1976a). « Note de lecture de N. Ruwet, Parallélismes et déviations en poésie ». Pratiques 10 , p. 105-112.

- (1976b). « Grammaire de texte - Théorie du discours - Narrativité ». Pratiques 11-12, p. 133-154.

- (1978). «Introduction aux problèmes de la cohérence des textes ». Langue française 38, p. 7-41.

- (1980). «Les formes directes et indirectes de l'argumentation ». Pratiques 28, p. 7-43.

- (1982). Études sur la cohérence et l'interprétation des discours. Thèse de doctorat d'état, Université de Franche-Comté.

- (1983). «Coherence as a Principle in the Interpretation of Discourse ». Text 3, 1, p. 71-99.

- (1987). « Les connecteurs et la portée de "selon" ». Revue Européenne des sciences sociales XXV, 77, p. $243-271$.

- (1988a). «Les plans d'organisation textuelle : périodes, chaînes, portées et séquences ».

Pratiques 57, p. 3-13.

- (1988b). « Les études sur la cohérence, la cohésion et la connexité textuelle depuis la fin des années 1960 ». Modèles linguistiques X-2, p. 45-66.

- (1989). « Analyse de discours, grammaire de texte et approche grammaticale des faits de textualité ». Le Français aujourd'hui 86, p. 12.

- (1990). «Connecteurs et portée. L'exemple de "se figurer que” ». In : Charolles, M., Fisher, S. \& Jayez, J. (éds), Le discours. Représentations et interprétations, Presses universitaires de Nancy, p. $147-166$

- (1993). « Les plans d'organisation du discours et leurs interactions ». In : Moirand, S. et al., 
Parcours linguistiques de discours spécialisés. Berne : Peter Lang, p. 301-314.

- (1997). «L'encadrement du discours. Univers, champs, domaines et espaces ». Cahier de recherche linguistique, 6, p. 1-73.

- (2001). « De la phrase au discours : quelles relations? ». In : Rousseau, A. (éd.), La sémantique des relations. Villeneuve d'Ascq : Presses universitaires du Septentrion, p. 237-260.

- (2002). La référence et les expressions référentielles en français. Paris, Ophrys.

- (2003). « De la topicalité des adverbiaux détachés en tête de phrase ». Travaux de linguistique 47, p. 11-51.

- (2011). « Cohérence et cohésion du discours ». In : Hölker, K. \& Marello, C. (Hrsg.), Dimensionen der Analyse Texten und Discursivent-Dimensioni dell'analisi di testi e discoursi. Münster : Lit Verlag, p. 153-173.

CHAROLles, M. \& COMBETTES, B. (1999). «Contribution pour une histoire récente de l'analyse du discours ». Langue française 121, p. 76-115.

combettes, B. (1977). «Ordre des éléments de la phrase et linguistique du texte ». Pratiques 13, p. 91-101.

- (1978). « Thématisation et progression thématique dans les récits d'enfants ». Langue Française 38 , p. $74-86$.

- (1983). Pour une grammaire textuelle. La progression thématique. Bruxelles : De Boeck/Duculot. - (1992). L'organisation du texte. Metz : Université Paul Verlaine-Metz/Publication du Centre d'analyse syntaxique.

- (1993). «Grammaire de phrase, grammaire de texte : le cas des progressions thématiques ». Pratiques 77, p. 43-57.

- (1998). Les constructions détachées. Paris : Ophrys.

COMBETTES, B. \& FRESSON, J. (1975). « Quelques éléments pour une linguistique textuelle ». Pratiques 6, p. 25-55.

COMBETTES, B. \& tOMASSONE, R. (1988). Le texte informatif, aspects linguistiques. Bruxelles : De Boeck. COQUET, J.-C. (1982). « L'École de Paris ». In : Coquet, J.-C., Sémiotique. L'École de Paris. Paris : Hachette, p. 5-64.

DANEŠ, F. (1974). « Functional Sentence Perspective and the Organization of the Text ». In : Daneš, F., Papers on Functional Sentence Perspective. Prague/La Haye : Academia/Mouton, p. 100-128.

DIJK, T. A. von (1972). « Aspects d'une Théorie Générative du Texte Poétique ». In : Greimas, A.-J. et al., Essais de sémiotique poétique. Paris : Larousse, p. 180-206.

- (1973a). « Modèles génératifs en théorie littéraire ». In : Bouazis, C. et al., Essais de la théorie du texte. Paris : Galilée, p. 79-99.

- (1973b). « Grammaires textuelles et structures narratives ». In : Chabrol, C. (éd.), Sémiotique narrative et textuelle. Paris : Larousse, p. 177-207.

- (1981-1982). «Attitudes et compréhension de textes ». Bulletin de psychologie 356, t. XXXV, p. 557-569.

DUCROT, O. (1980). Les mots du discours. Paris : Minuit.

FIRBAS, J. (1964). « On Defining the Theme in Functional Sentence Perspective Analysis ». Travaux Linguistiques de Prague 1, p. 267-280.

FouCAult, M. (1969). L'Archéologie du savoir. Paris : Gallimard.

FUCHS, C. (1985). «L'ambiguïté et la paraphrase, propriétés fondamentales des langues naturelles ». In Fuchs C. (dir.), Aspects de l'ambiguité et de la paraphrase dans les langues naturelles. Berne : Peter Lang, p. 7-35. 
GARDES TAMINE, J. \& PELLIZZA, M.-A. (1998). La construction du texte. De la grammaire au style. Paris : Armand Colin.

GIVÓN, T. (1998). « L'approche fonctionnelle de la grammaire ». Verbum 20, 3, p. 257-288.

GOLDENSTEIn, J.-P. (1976). « Une grammaire de texte pour la composition française ». Pratiques 10, p. 69-79.

GREISCH, J. (éd.) (1987). Le texte comme objet philosophique. Paris : Beauchesne.

GREIMAS, A. J. (1966). Sémantique structurale. Paris : Larousse.

- (1970). Du Sens. Paris : Seuil.

- (1976a). Maupassant. La sémiotique du texte : exercices pratiques. Paris : Seuil.

- (entretien avec) (1976b). "Sémiotique narrative et textuelle ». Pratiques 11-12, p. 5-12.

GRICE, H. P. (1975). « Logic and conversation ». In : Cole, P. \& Morgan, J. L. (eds.), Syntax and

Semantics 3 : Speech Acts. New York : Academic Press, p. 41-58.

HALTÉ, J.-F. (1974). « La grammaire et les textes ». Pratiques 3-4, p. 45-59.

HALTÉ, J.-F. \& PETITJEAN, A. (1978). « Lire et écrire en situation scolaire ». Langue française 38, p. 58-73.

HARRIS, Z. S. (1952). « Discourse Analysis ». Language 28-1, p. 1-30.

HJELMSLEV, L. T. (1971) [1968]. Prolégomènes à une théorie du langage. Paris : Minuit.

JACQUES, F. (2002). De la textualité. Pour une textologie générale et comparée. Paris : Maisonneuve. - (2007). L'arbre du texte et ses possibles. Paris : Vrin.

KINTSCH, W. (1981-1982). « Aspects de la compréhension de textes ». Bulletin de psychologie 356, t. XXXV, p. 777-787.

KINTSCH, W. \& VAN DIJK, T. A. (1975). « Comment on se rappelle et on résume des histoires ». Langages 40 , p. 98-116.

- (1984) [1978]. «Vers un modèle de la compréhension et de la production de textes ». In :

Denhière, G. (éd.), Il était une fois... Compréhension et souvenir de récits. Lille : Presses de l'université de Lille, p. 85-142.

LANG, E. (1972). « Quand une "grammaire de texte" est-elle plus adéquate qu'une "grammaire de phrase" ? ", Langages 26, p. 75-80.

LEGALLOIS, D. (2006). « Des phrases entre elles à l'unité réticulaire du texte ». Langages 163, p. 56-70.

LUNDQUIST, L. (1980). La Cohérence textuelle : syntaxe, sémantique, pragmatique. Copenhague : Nyt Nordisk Forlag Arnold Busck.

- (1999). «Le Factum Textus : fait de grammaire, fait de linguistique ou fait de cognition?». Langue française 121 , p. 56-75.

MAINGUENEAU, D. (1976). Initiation aux méthodes de l'analyse du discours. Paris : Hachette.

- (1984). Genèses du discours. Bruxelles : Mardaga.

- (1987). Nouvelles tendances en analyse du discours. Paris : Hachette.

- (1991) [1997]. L'analyse du discours. Introduction aux lectures de l'archive. Paris : Hachette.

- (2014). Discours et analyse de discours. Paris : Armand Colin.

MAingueneau, D. \& Charaudeau, P. (éds) (2002). Dictionnaire d'analyse du discours. Paris : Seuil.

MOESCHLER, J. \& REBOUL, A. (1998). La pragmatique aujourd'hui. Paris : Seuil. 
MOIRAND, S. et al. (éds) (1993). Parcours linguistiques de discours spécialisés. Berne : Peter Lang.

PAVEAU, M.-A. \& SARFATI, G.-E. (2003). Les grandes théories de la linguistique. Paris : Armand Colin. PÊCHEUX, M. (1969). L'analyse automatique du discours. Paris : Dunod.

- (1990). L'inquiétude du discours, textes choisis et présentés par Denise Maldidier. Paris : Éditions des Cendres.

PÊCHEUX, M. \& FUCHS, C. (1975). « La sémantique et la coupure saussurienne : langue, langage,

discours ». Langages 37 , p. 7-80.

Provost-CHAUVeau, G. (1971). « Problèmes théoriques et méthodologiques en analyse du

discours ». Langue française 9, p. 6-21.

RASTIER, F. (1989). Sens et textualité. Paris : Hachette.

- (2001). Arts et sciences du texte. Paris : Presses universitaires de France.

RICœUR, P. (1986). Du texte à l'action. Essais d'herméneutique II. Paris : Seuil.

RIEGEL, M., PeLlat, J.-C. \& RIOUL, R. (1994). Grammaire méthodique du français. Paris : Presses universitaires de France.

RÜCK, H. (1980) [1978]. Linguistique textuelle et enseignement du français. Trad. de l'allemand par H. Rück. Paris : Hatier-Credif.

RUWET, N. (1975). «Parallélismes et déviations en poésie ». In : Kristeva, J., Milner, J.-C. \& Ruwet, N. (dirs), Langue, discours, société. Pour Émile Benveniste. Paris : Seuil, p. 307-351.

SAUSSURE, F. de (2002). Écrits de linguistique générale. Paris : Gallimard.

SIMONIN-GRUMBACH, J. (1975). « Pour une typologie des discours ». In : Kristeva, J., Milner, J.-C. \& Ruwet, N. (dirs), Langue, discours, société. Pour Émile Benveniste. Paris : Seuil, p. 85-121.

- (1977). « Linguistique textuelle et étude des textes littéraires : à propos de Le Temps de $\mathrm{H}$.

Weinrich ». Pratiques 13, p. 77-90.

SLAKTA, D. (1975). « L'ordre du texte ». Études de linguistique appliquée 19, p. 30-42.

SOUTET, O. (2005) [1995]. Linguistique. Paris : Presses universitaires de France.

SPERBER, D. \& WILSON, D. (1986). Relevance. Communication and cognition. Oxford : Basil Blackwell.

STATI, S. (1990). Le transphrastique. Paris : Presses universitaires de France.

SWALES, J. (1990). Genre Analysis. English in Academic and Research Settings. Cambridge : Cambridge university Press.

TODOROV, T. (1973). Poétique. Qu'est-ce que le structuralisme ?-2. Paris : Seuil

- (1975). « La notion de littérature ». In : Kristeva, J., Milner, J.-C. \& Ruwet, N. (éds), Langue,

discours, société. Paris : Seuil, p. 352-364.

- (1978). Les genres de discours. Paris : Seuil.

VIPREY, J.-M. (2006). « Structure non séquentielle des textes ». Langages 163, p. 71-85.

WEINRICH, H. (1994) [1964-1971]. Tempus. Stuttgart-Belin-Köln : Kohlhammer.

\section{RÉSUMÉS}

Cet article a pour but de contextualiser les travaux menés dans Pratiques dans le domaine de la théorie et de la pratique des textes et des discours. Ces pages reviennent sur le bouillonnement 
intellectuel des années 60-70 et la richesse du dialogue mené pendant près de 40 ans à l'intérieur et en dehors du collectif. Contribuant au développement d'une linguistique textuelle en langue française, la revue a joué un rôle de diffusion d'idées et de recherches qui n'étaient alors pas académiquement reconnues. Elle a joué aussi un rôle de stimulation de questionnements en prise sur la demande sociale de l'école. Cette linguistique textuelle "à la française " apparait aujourd'hui comme doublement axée sur la question théorique des problèmes du texte et sur la collaboration avec les champs disciplinaires et domaines d'application proches que sont l'analyse de discours, la stylistique et la traduction, mais aussi en prise sur les didactiques de l'écrit et de la lecture des textes.

This article contextualise the works led in Pratiques in the field of the theory and of the practice of texts and speeches. These pages return on the intellectual bubbling of the years 1960-70 and the wealth of the dialogue led during almost 40 years. Contributing to the development of a text linguistics in French language, Pratiques played a role of broadcasting of ideas and researches which were not recognized then academically. The revue also played a seminal role of questionings in grip on the social request of the school. This "French-style" text linguistics appears as double centred on the theoretical question of the problems of the text and on the collaboration with the disciplinary fields and the close fields of application as discourse analysis, stylistics and translation, but also in grip on the didactics of texts.

\section{INDEX}

Mots-clés : linguistique textuelle, théorie du texte, genres de discours, Émile Benveniste, Roland Barthes, Algirdas J. Greimas, Tzvetan Todorov, Pratiques

Keywords : text linguistics, texttheory, genres of discourse, Émile Benveniste, Roland Barthes, Algirdas J. Greimas, Tzvetan Todorov, Pratiques

\section{AUTEUR}

JEAN-MICHEL ADAM 